\title{
Translocator Protein Localises To CD11b+ Macrophages In Atherosclerosis
}

Chantal Kopecky ${ }^{1}$, Elvis Pandzic ${ }^{2}$, Arvind Parmar $^{3}$, Jeremy Szajer ${ }^{4}$, Victoria Lee ${ }^{1}$, Alexander Dupuy ${ }^{1}$, Andrew Arthur ${ }^{3}$, Sandra Fok ${ }^{2}$, Renee Whan ${ }^{2}$, William J. Ryder ${ }^{4 \star}$, Kerry-Anne Rye ${ }^{1}$ and Blake J. Cochran ${ }^{1}$

1 School of Medical Sciences, Faculty of Medicine, UNSW Sydney, Sydney, Australia

2 Biomedical Imaging Facility, Mark Wainwright Analytical Centre, UNSW Sydney, Sydney, Australia

3 Australian Nuclear Science and Technology Organisation, Sydney, Australia

4 Department of Nuclear Medicine, Concord Repatriation General Hospital, Sydney, Australia

Current location: *Health Education and Training Institute, Sydney, NSW, Australia 3948 words

Corresponding author:

Dr Blake J. Cochran

School of Medical Sciences

Faculty of Medicine

UNSW Sydney 2052

NSW, Australia

b.cochran@unsw.edu.au 
Background: Atherosclerosis is characterized by lipid deposition, monocyte infiltration and foam cell formation in the artery wall. Translocator protein (TSPO) is abundantly expressed in lipid rich tissues. Recently, TSPO has been identified as a potential diagnostic tool in cardiovascular disease. The purpose of this study was to determine if the TSPO ligand, ${ }^{18} \mathrm{~F}$-PBR111, can identify early atherosclerotic lesions and if TSPO expression can be used to identify distinct macrophage populations during lesion progression.

Methods and Results: ApoE ${ }^{-/}$mice were maintained on a high-fat diet for 3 or 12 weeks. C57BL/6J mice maintained on chow diet served as controls. Mice were administered ${ }^{18}$ F-PBR111 intravenously and PET/CT imaged. After euthanasia, aortas were isolated, fixed and optically cleared. Cleared aortas were immunostained with DAPI, and fluorescently labelled with antibodies to-TSPO, the tissue resident macrophage marker F4/80 and the monocyte-derived macrophage marker CD11b. TSPO expression and the macrophage markers were visualised in fatty streaks and mature lesions by light sheet microscopy. While tissue resident $\mathrm{F} 4 / 80^{+}$macrophages were evident in the arteries of animals without atherosclerosis, no $\mathrm{CD}_{11} \mathrm{~b}^{+}$macrophages were observed in these animals. In contrast, mature plaques had high CD11b and low F4/80 expression. A 3-fold increase in the uptake of ${ }^{18}$ F-PBR111 was observed in the aortas of atherosclerotic mice relative to controls.

Conclusions: Imaging of TSPO expression is a new approach for studying atherosclerotic lesion progression and inflammatory cell infiltration. The TSPO ligand, ${ }^{18}$ F-PBR111, is a potential clinical diagnostic tool for the detection and quantification of atherosclerotic lesion progression in humans. 


\section{Introduction}

Atherosclerosis, a chronic inflammatory disease characterized by accumulation of lipids and inflammatory cells such as macrophage foam cells within the arterial wall, is a leading cause of death globally ${ }^{1}$. There is significant debate as to the relative contributions of tissue-resident versus monocyte-derived macrophages to atherosclerotic lesion progression ${ }^{2}$.

Atherosclerotic progression commonly develops as an indolent process over decades $^{3}$, making early identification of the condition challenging. Clinical assessment of atherosclerosis currently involves either invasive angiography or intravascular ultrasound (IVUS), or is limited to recognition of advanced disease by, for example, calcium scoring ${ }^{4}$. Recent efforts have focused on establishing dynamic imaging approaches to identify clinically relevant atherosclerosis. The development, assessment and broad adoption of novel PET/CT techniques enables early detection and diagnosis of cardiovascular disease ${ }^{5}$. Non-invasive PET imaging, using specifically targeted radiolabelled probes to track active atherosclerosis may provide a highly suitable method to identify and further understand atherogenesis ${ }^{6}$. In this regard, translocator protein (TSPO) has recently been identified as a therapeutic target and diagnostic tool in cardiovascular disease ${ }^{7}$.

TSPO is an $18 \mathrm{kDa}$ mitochondrial membrane protein that is involved in steroidogenesis and cholesterol transport ${ }^{8}$. It is expressed abundantly throughout the body, and is highly upregulated in microglia and macrophages during inflammation ${ }^{9}$ 10. Clinically, the TSPO radioligand ${ }^{11} \mathrm{C}-\mathrm{PK} 11195$ has been used to image atherosclerotic lesions in the carotid artery ${ }^{11}$. However, use of this ligand was limited 
due to uptake by healthy vessels. Newer TSPO ligands have been developed in recent years and ${ }^{18} \mathrm{~F}$-PBR111 has evolved as promising candidate owing to its higher affinity and binding specificity compared to ${ }^{11} \mathrm{C}-\mathrm{PK} 11195^{12}$. Moreover, as TSPO is involved in the regulation of cardiovascular, atherosclerotic and inflammatory processes, TSPO ligands are promising therapeutic and diagnostic tools for use in cardiovascular disease ${ }^{7}$. Treating macrophages with TSPO agonists has been shown to increase cholesterol efflux ${ }^{13}$ and treatment of cholesterol fed guinea pigs and $\mathrm{apoE}^{-/}$mice with a peptide containing the cholesterol binding domain of TSPO reduced serum cholesterol levels and decreased atheroma formation $^{14}$.

In this study, the ability of ${ }^{18} \mathrm{~F}$-PBR111 to detect atherosclerosis progression in vivo was examined. Specifically, the ability of ${ }^{18} \mathrm{~F}-\mathrm{PBR} 111$ to detect atherosclerotic lesions at different stages of development was investigated. It also asked if TSPO expression increases with disease progression and if distinct macrophage populations can be distinguished by TSPO expression during lesion development. The findings of this study provide a rationale for the development of TSPO radioligands as clinical diagnostic tools for the detection and quantification of atherosclerosis in humans by non-invasive, biomedical imaging. 


\section{Methods}

Mice. C57BL/6J and B6.129P2-Apoe $e^{t m 1 U n c}\left(\mathrm{apoE}^{-/-}\right)$mice were obtained from Animal Resources Centre (Perth, Australia) and maintained in pathogen-free conditions at the Brain and Mind Centre, University of Sydney. Control C57BL/6J mice were fed a standard chow diet, whilst apoE ${ }^{-/}$mice were maintained on a high fat diet (HFD; $22 \%$ fat, $0.15 \%$ cholesterol, Specialty Feeds, Perth, Australia) for the time periods stated. All procedures were approved by the University of Sydney Animal Ethics Committee and followed the NIH Guide for the care and use of laboratory animals, Eighth edition (2011).

Light sheet microscopy. Mice were euthanized after PET/CT scanning and aortas were isolated and fixed overnight in $4 \%(\mathrm{v} / \mathrm{v})$ paraformaldehyde. Lipid was cleared from the tissue as previously described ${ }^{15}$. Briefly, fixed tissues were incubated in Reagent 1 (25\% (w/w) urea, 25\% (w/w) Quadrol, 15\% (w/w), Triton X-100) for $16 \mathrm{~h}$ at $37^{\circ} \mathrm{C}$ and then washed for $36 \mathrm{~h}$ in PBS. Optically cleared samples were then stained with Alexa 488 labelled anti-TSPO antibody (1:500, Abcam), Alexa 647 labelled anti-F4/80 antibody (1:200, Abcam), PE/Dazzle 594 labelled anti-CD11b antibody (1:200, Biolegend) and DAPI (ThermoFisher) in PBS plus $0.1 \%(w / v)$ BSA, $0.3 \%(v / v)$ Triton $\mathrm{X}-100$ for $16 \mathrm{~h}$ at $37^{\circ} \mathrm{C}$. Samples were washed for $36 \mathrm{~h}$ in PBS and incubated in refractive index matched Reagent $2(25 \%(w / w)$ urea, $50 \%(w / w)$ sucrose, $10 \%(w / w)$ triethanolamine) for 2 days. Stained tissues were imaged by lightsheet fluorescence microscopy (Zeiss Lightsheet Z.1; Carl Zeiss) housed at UNSW Sydney (Biomedical Imaging Facility, Mark Wainwright Analytical Centre, UNSW Sydney). The samples were placed in the imaging chamber filled with Reagent 2 and imaging was performed using a $5 \times / 0.16$ detection lens and $5 \times / 0.1$ 
illumination lens. The four fluorescent signatures were collected as follows: DAPI $405 \mathrm{~nm}$ excitation, 460-500 nm emission; TSPO $488 \mathrm{~nm}$ excitation, 505-545 nm emission; CD11b $561 \mathrm{~nm}$ excitation, 575-615 nm emission and F4/80 $638 \mathrm{~nm}$ excitation, 660/LP nm emission. To capture the entire sample, a Z stack of $\sim 1000$ frames and $\sim 3 \times 4$ tiles were captured, at $30 \mathrm{~ms}$ exposure on two CMOS cameras with $1920 \times 1920$ pixel images and $2.329 \mu \mathrm{m}$ pixel size. Z-stack images were collected with a mean optimal sectioning step of $4 \mu \mathrm{m}$. Images were processed, stitched, rendered and visualized in 4D with Arivis Vision4D software (Arivis).

Data quantification and image analysis. Stitched data was exported to .tif files for further processing in Matlab 2018a (Mathworks). When imported, data from each channel was registered to align with the DAPI channel in order to correct potential drift artefacts from imaging. Following alignment, TSPO channel data was imported into Microscopy Image Browser ${ }^{16}$ running in Matlab for segmentation of TSPO enriched regions and the aortic wall in each optical section. TSPO 3D masks were then assessed for $\mathrm{TSPO}^{+}$volume, while aorta wall masks were used to calculate total aorta and aortic wall volumes. Using these masks, the standard deviation of TSPO channel intensity within the aorta wall was determined, and the ratio of standard deviation and average intensity calculated for each slice. In order to characterize the amount of cross correlation between TSPO and CD11b, image cross correlation spectroscopy was used ${ }^{17}$. The $\mathrm{TSPO}^{+}$and aorta wall masks described above were applied to the TSPO and CD11b channels in each slice to exclude pixels outside of the mask from the cross correlation function. The cross correlation function was characterized for each slice by fitting to a 2D Gaussian 
function and the amplitude extracted, with higher amplitude indicating higher crosscorrelation between TSPO and CD11b.

PET study. Mice were maintained on chow or high fat diet as indicated. Mice were anaesthetised with isoflurane administered via precision vaporiser (induction, 4\%; maintenance, 1.5-2\%), and maintained in a surgical plane of anaesthesia for the duration of the procedure. After positioning the mice in the gantry of an Inveon PET/CT Scanner (Siemens, Munich, Germany), a 60 min PET scan commenced immediately after injection of ${ }^{18} \mathrm{~F}$-PBR111 (8-18 MBq, $\left.100 \mu \mathrm{L}, 0.2 \mathrm{nM}\right)$ via the lateral tail vein. To allow for co-registration of radiotracer uptake with the aorta, a CT scan was performed immediately after the PET scan.

Image reconstruction. Image reconstruction was performed using IAW 2.02 (Siemens). The listmode data was histogrammed into 16 frames $(6 \times 10 \mathrm{~s}, 4 \times 60 \mathrm{~s}$, $1 \times 300 \mathrm{~s}, 5 \times 600 \mathrm{~s})$ for the period $0-60 \mathrm{~min}$ after tracer injection. Emission sinograms were reconstructed using 2D-filter back projection with a zoom 1.5. The reconstructed images consisted of 16 frames of a $128 \times 128 \times 159$ matrix with a voxel size of $0.52 \times 0.52 \times 0.796 \mathrm{~mm}^{3}$, corrected for attenuation (CT-based), scatter, randoms, normalisation, isotope decay, branching ratio, deadtime and were calibrated to $\mathrm{Bq} / \mathrm{mL}$.

Region of interest. The thoracic aorta, from the commencement of the descending aorta to the aortic hiatus of the diaphragm, was used as the relevant region of interest $(\mathrm{ROI})$ for quantification. The thoracic aorta rather than the aortic arch was chosen because it did not require cardiac gating. The ROI for each mouse was 
demarcated using a voxel size of $0.2 \times 0.2 \times 0.2 \mathrm{~mm}^{3}$ by a physician trained in medical imaging interpretation. ROls were delineated using the CT images viewed using AMIDE software ${ }^{18}$ on a 4-megapixel EIZO monitor at the Department of Nuclear Medicine, Concord Hospital, NSW. The interpreting physician was blinded to the identity of each mouse. Each ROI was independently reviewed by two additional interpreters to ensure accurate demarcation of the thoracic aorta. Once the ROls were agreed upon, the PET images where used to demarcate a secondary ROI within the lateral tail vein, at the base of the tail. This second ROI represented the level of ${ }^{18} \mathrm{~F}$-PBR111 in the blood, rostral to the radiopharmaceutical injection site.

Statistical analysis. All data presented are mean \pm SD. Data was analysed using Prism 7 (GraphPad). One-way ANOVA or Kruskal-Wallis tests, as appropriate, were used to determine statistically significant differences between data sets. A p value $<0.05$ was considered statistically significant. 


\section{Results}

\section{TSPO accumulates in atherosclerotic lesions}

Aortas were rendered optically clear (Fig. 1A) and stained with fluorescently labelled TSPO and DAPI antibodies. Expression of TSPO during atherosclerotic lesion development was determined in the cleared and immunostained aortas by 3D imaging using lightsheet fluorescence microscopy. TSPO expression was not observed in the aortas of control mice (Fig. 1B), and was restricted to the vessel wall of aortas of 3-week HFD fed apoE ${ }^{-/-}$mice (Fig. 1C). 12-week HFD fed apoE ${ }^{-/-}$mice showed high TSPO expression in both regions of mature plaque and fatty streaks lining the vessel wall (Fig. 1D). Quantification of $\mathrm{TSPO}^{+}$volume demonstrated a significant increase in 3-week HFD fed $\mathrm{apoE}^{-/-}$mice compared to controls $\left(0.64 \pm 0.23 \%\right.$ vs $0.04 \pm 0.06 \% \mathrm{TSPO}^{+}$volume for 3 -week HFD and control, respectively; $p<0.05) .12$ weeks of HFD led to a further significant increase in $\mathrm{TSPO}^{+}$ volume $\left(34.05 \pm 24.26 \% \mathrm{TSPO}^{+}\right.$volume; $\mathrm{p}<0.05$ vs both control and 3 -week groups) (Fig. 1E).

To better characterise the pattern of TSPO expression in the aortas of atherosclerotic mice, a masking algorithm was applied (Fig. 2A) to determine a pixelbased intensiometric analysis on a per plane basis, and then calculated the standard deviation (SD) of the TSPO channel intensity within this pre-defined region of interest. These results show that the SD of the TSPO signal in the 12-week HFD fed $\operatorname{apoE}^{-/-}$mice was significantly increased relative to both control and 3-week HFD mice ( $p<0.05$ vs both) (Fig. 2B). 


\section{TSPO is preferentially co-localized to CD11b+ macrophages}

To investigate the presence of specific macrophage subpopulations throughout atherosclerotic lesion development, aortas were immunostained with the macrophage markers CD11b and F4/80. In aortas from control C57BL/6J mice (Fig. 3A) and 3-week HFD fed apoE ${ }^{-/-}$mice (Fig. 3B), F4/80+ macrophages predominated, while CD11b staining was minimal. In contrast, significant CD11b staining was observed in the aortas of 12-week HFD fed apoE ${ }^{-/-}$mice (Fig. 3C). Co-localization of the respective macrophage markers and TSPO was determined by calculating the spatial image cross-correlation (ICCS) and expressing the results as the change in ICCS (TSPO/CD11b - TSPO/F4/80) (Figure 3D). High ICCS values, which are indicative of a high correlation between CD11b and TSPO levels, were observed in mature plaque (Fig. 3E) and fatty streak (Fig. 3F) regions. TSPO expression was associated with CD11b expression, as evidenced by the positive ICCS values observed in the vast majority of slices.

\section{${ }^{18}$ F-PBR111 detects mature, but not early lesions in vivo}

Given the level of expression and localisation of TSPO in the aortas of apoE ${ }^{-/-}$mice maintained on HFD, it was next asked if the TSPO agonist ${ }^{18} \mathrm{~F}-\mathrm{PBR} 111$ could also detect atherosclerosis in these mice in vivo. To control for the level of circulating ${ }^{18} \mathrm{~F}$ PBR111, activity in the lateral tail vein was subtracted from the region of interest in the aorta (Fig. 4). This allowed for the tissue specific uptake in the aorta to be measured. Uptake of ${ }^{18}$ F-PBR111 in the aorta was significantly increased in 12-week high fat fed apoE ${ }^{-/-}$mice compared to both 3-week HFD apoE ${ }^{-/-}$and wildtype control mice (maximal activity at $2.7 \mathrm{~min}$ post injection; $9.15 \pm 3.60$ vs $0.77 \pm 1.32$ and $1.22 \pm 1.20 \% \mathrm{ID} / \mathrm{cm}^{3}$, for 12-week HFD, 3-week HFD and control mice, respectively; 
$\mathrm{p}<0.05)$ (Fig. 5A). Aortic specific levels of ${ }^{18} \mathrm{~F}-\mathrm{PBR} 111$ were significantly increased in 12-week HFD apoE ${ }^{-/-}$mice over the course of data acquisition (incremental area under the curve: $4015 \pm 2808$ vs $90 \pm 509$ and $443 \pm 474$ for 12 -week HFD, 3 -week HFD and control mice, respectively; $p<0.05$ for 12-week HFD vs both) (Fig. 5B). 


\section{Discussion}

This study found that TSPO expression in aortas of high-fat fed apoE ${ }^{-/-}$mice was progressively elevated with atherosclerotic lesion progression. These results were validated by PET/CT with increased uptake of the TSPO radioligand ${ }^{18} \mathrm{~F}-\mathrm{PBR} 111$ in mice with advanced atherosclerosis relative to mice with early stage disease and control mice without disease. It was also demonstrated that macrophage subpopulations differed between early atherosclerosis and more mature plaque regions. Whilst $\mathrm{F} 4 / 80^{+}$tissue resident macrophages were evident in aortas of animals without atherosclerosis, no CD11b staining was observed in the arteries of these mice. In contrast, atherosclerotic lesions had significantly increased CD11b expression that co-localized with TSPO.

Atherosclerosis is a leading cause of death globally and is now widely recognized as a lipid-driven, systemic inflammatory disease ${ }^{19}$. A critical initiating event in atherosclerosis is excessive lipid accumulation and foam cell formation within the artery wall, leading to activation of inflammatory signaling ${ }^{20}$. Macrophages and monocytes are the major immune cells in atherosclerosis and play important roles throughout all stages of lesion progression ${ }^{21}$. However, macrophage development is a highly heterogeneous process, and embryonically derived tissue resident macrophages can be joined or replaced by recruited monocytes upon inflammatory stimuli $^{19,22}$.

This study has shown that $\mathrm{CD} 1 \mathrm{~b}^{+}$macrophages predominate in advanced atherosclerotic lesions. Contrastingly, no CD11b staining was present and F4/80+ regions were evenly distributed throughout the aortas of control C57BL/6J mice and 
early lesions in 3 weeks HFD-fed apoE ${ }^{-/-}$mice. Thus, this data shows for the first time that TSPO accumulates in the same regions of atherosclerotic lesions as specific macrophage subpopulations. There is significant debate regarding the utility and specificity of markers to determine the lineage and source of the macrophages in vivo ${ }^{23}$. CD11b is expressed by newly migrating macrophages in the $\operatorname{liver}^{24,25}$, whilst F4/80 is present on the surface of tissue resident macrophages in the spleen and liver ${ }^{24}$.

A challenge in clinical management is that most patients with atherosclerosis do not display overt disease-related symptoms. This also presents a substantial diagnostic challenge $^{26}$. Recent developments have focused on a shift to novel, non-invasive techniques such as PET, coupled with $\mathrm{CT}$ or MRI to allow precise anatomical localization and characterisation of atherosclerotic lesions ${ }^{6}$. In agreement with previous studies, the results presented here demonstrate that TSPO may be a useful marker of atherosclerotic lesion development. Furthermore, the specific association of TSPO with CD11b warrants further investigation and may provide insights into plaque development, specifically relating to macrophage infiltration of the vessel wall.

In conclusion, this study provides evidence of the diagnostic potential of TSPO imaging as a means of studying atherosclerotic lesion progression. These results show TSPO expression in a distinct macrophage population that plays an important role in atherosclerotic lesion progression. There is thus a significant rationale for the development and use of radiolabelled TSPO ligands as tools for the detection and quantification of atherosclerotic lesion progression. 
Acknowledgements This work was supported by an AINSE Research Award to BJC. The authors acknowledge the facilities, scientific and technical assistance of the National Imaging Facility, at the ANSTO-BMC imaging platform, University of Sydney. 


\section{References}

1. Libby P. Inflammation in atherosclerosis. Arteriosclerosis, thrombosis, and vascular biology. 2012;32:2045-51.

2. Rahman MS, Murphy AJ and Woollard KJ. Effects of dyslipidaemia on monocyte production and function in cardiovascular disease. Nat Rev Cardiol. $2017 ; 14: 387-400$.

3. Tarkin JM, Dweck MR, Evans NR, Takx RA, Brown AJ, Tawakol A, Fayad ZA and Rudd JH. Imaging Atherosclerosis. Circ Res. 2016;118:750-69.

4. Owen DR, Lindsay AC, Choudhury RP and Fayad ZA. Imaging of atherosclerosis. Annual review of medicine. 2011;62:25-40.

5. Evans NR, Tarkin JM, Chowdhury MM, Warburton EA and Rudd JH. PET Imaging of Atherosclerotic Disease: Advancing Plaque Assessment from Anatomy to Pathophysiology. Curr Atheroscler Rep. 2016;18:30.

6. Tarkin JM, Joshi FR, Rajani NK and Rudd JH. PET imaging of atherosclerosis. Future Cardiol. 2015;11:115-31.

7. Qi X, Xu J, Wang F and Xiao J. Translocator protein (18 kDa): a promising therapeutic target and diagnostic tool for cardiovascular diseases. Oxidative medicine and cellular longevity. 2012;2012:162934.

8. Selvaraj $\mathrm{V}$ and Stocco DM. The changing landscape in translocator protein (TSPO) function. Trends Endocrinol Metab. 2015;26:341-8.

9. Owen DR, Narayan N, Wells L, Healy L, Smyth E, Rabiner EA, Galloway D, Williams JB, Lehr J, Mandhair H, Peferoen LA, Taylor PC, Amor S, Antel JP, Matthews PM and Moore CS. Pro-inflammatory activation of primary microglia and macrophages increases $18 \mathrm{kDa}$ translocator protein expression in rodents but not humans. J Cereb Blood Flow Metab. 2017;37:2679-2690. 
10. Batarseh A and Papadopoulos V. Regulation of translocator protein $18 \mathrm{kDa}$ (TSPO) expression in health and disease states. Mol Cell Endocrinol. 2010;327:1-

12.

11. Gaemperli O, Shalhoub J, Owen DR, Lamare F, Johansson S, Fouladi N, Davies $\mathrm{AH}$, Rimoldi OE and Camici PG. Imaging intraplaque inflammation in carotid atherosclerosis with 11C-PK11195 positron emission tomography/computed tomography. Eur Heart J. 2012;33:1902-10.

12. Frédéric D, Françoise $\mathrm{H}$, Annelaure D, Bertrand K, Christopher F, Tien P, Bertrand T and Andrew K. Radiosynthesis of [18F]PBR111, a selective radioligand for imaging the translocator protein $(18 \square \mathrm{kDa})$ with PET. Journal of Labelled Compounds and Radiopharmaceuticals. 2008;51:435-439.

13. Taylor JM, Allen AM and Graham A. Targeting mitochondrial $18 \mathrm{kDa}$ translocator protein (TSPO) regulates macrophage cholesterol efflux and lipid phenotype. Clin Sci (Lond). 2014;127:603-13.

14. Lecanu L, Yao ZX, McCourty A, Sidahmed el K, Orellana ME, Burnier MN and Papadopoulos V. Control of hypercholesterolemia and atherosclerosis using the cholesterol recognition/interaction amino acid sequence of the translocator protein TSPO. Steroids. 2013;78:137-46.

15. Susaki EA, Tainaka K, Perrin D, Yukinaga $H$, Kuno A and Ueda HR. Advanced CUBIC protocols for whole-brain and whole-body clearing and imaging. Nature protocols. 2015;10:1709-27.

16. Belevich I, Joensuu M, Kumar D, Vihinen H and Jokitalo E. Microscopy Image Browser: A Platform for Segmentation and Analysis of Multidimensional Datasets. PLoS Biol. 2016;14:e1002340. 
17. Comeau JW, Kolin DL and Wiseman PW. Accurate measurements of protein interactions in cells via improved spatial image cross-correlation spectroscopy. Mol Biosyst. 2008;4:672-85.

18. Loening AM and Gambhir SS. AMIDE: a free software tool for multimodality medical image analysis. Molecular imaging. 2003;2:131-7.

19. Swirski FK, Robbins CS and Nahrendorf M. Development and Function of Arterial and Cardiac Macrophages. Trends in immunology. 2016;37:32-40.

20. Robbins CS, Hilgendorf I, Weber GF, Theurl I, Iwamoto Y, Figueiredo JL, Gorbatov R, Sukhova GK, Gerhardt LM, Smyth D, Zavitz CC, Shikatani EA, Parsons M, van Rooijen N, Lin HY, Husain M, Libby P, Nahrendorf M, Weissleder R and Swirski FK. Local proliferation dominates lesional macrophage accumulation in atherosclerosis. Nat Med. 2013;19:1166-72.

21. Ley $\mathrm{K}$, Miller $\mathrm{YI}$ and Hedrick CC. Monocyte and macrophage dynamics during atherogenesis. Arterioscler Thromb Vasc Biol. 2011;31:1506-16.

22. Honold L and Nahrendorf M. Resident and Monocyte-Derived Macrophages in Cardiovascular Disease. Circ Res. 2018;122:113-127.

23. Hume DA. Differentiation and heterogeneity in the mononuclear phagocyte system. Mucosal immunology. 2008;1:432-41.

24. Lloyd CM, Phillips AR, Cooper GJ and Dunbar PR. Three-colour fluorescence immunohistochemistry reveals the diversity of cells staining for macrophage markers in murine spleen and liver. J Immunol Methods. 2008;334:70-81.

25. Morinaga H, Mayoral R, Heinrichsdorff J, Osborn O, Franck N, Hah N, Walenta E, Bandyopadhyay G, Pessentheiner AR, Chi TJ, Chung H, Bogner-Strauss JG, Evans RM, Olefsky JM and Oh DY. Characterization of distinct subpopulations of hepatic macrophages in HFD/obese mice. Diabetes. 2015;64:1120-30. 
26. Go AS, Mozaffarian D, Roger VL, Benjamin EJ, Berry JD, Blaha MJ, Dai S, Ford ES, Fox CS, Franco S, Fullerton HJ, Gillespie C, Hailpern SM, Heit JA, Howard VJ, Huffman MD, Judd SE, Kissela BM, Kittner SJ, Lackland DT, Lichtman JH, Lisabeth LD, Mackey RH, Magid DJ, Marcus GM, Marelli A, Matchar DB, McGuire DK, Mohler ER, 3rd, Moy CS, Mussolino ME, Neumar RW, Nichol G, Pandey DK, Paynter NP, Reeves MJ, Sorlie PD, Stein J, Towfighi A, Turan TN, Virani SS, Wong ND, Woo D and Turner MB. Heart disease and stroke statistics--2014 update: a report from the American Heart Association. Circulation. 2014;129:e28-e292. 


\section{Figure Legends}

Figure 1: TSPO expression increases with atherosclerosis progression. (A) Isolated aorta before (left) and after (right) optical clearing; Representative 3D reconstructed images of aortas isolated from (B) C57BL/6J or apoE ${ }^{-/-}$mice after (C) 3- and (D) 12-weeks on HFD. TSPO expression is indicated in yellow, DAPI is indicated in blue. (E) Quantification of TSPO+ volume in aortas. Values are mean \pm SD. $n=4 /$ group, ${ }^{*} p<0.05$. Grid in $(A)=1 \mathrm{~cm}^{2}$.

Figure 2: Quantification of the variability of intravascular TSPO expression. (A) Representative images before and after a masking algorithm was applied to generate a region of interest and measure TSPO channel intensity within the mask. (B) SD of TSPO signal within the mask normalized to TSPO signal in aorta wall. Each dot represents the mean TSPO intensity per plane normalized to the mean overall TSPO intensity of each sample. Red bars indicate means and blue regions indicate confidence intervals. ${ }^{* * *} \mathrm{p}<0.001$

Figure 3: TSPO expression correlates with CD11b. Expression of the macrophage markers $\mathrm{CD} 11 \mathrm{~b}$ and $\mathrm{F} 4 / 80$ in aortas from (A) chow fed wildtype controls (B) 3-week HFD and (C) 12-week HFD fed apoE ${ }^{-/-}$mice. (D) To quantify colocalization between the macrophage markers CD11b and F4/80 with TSPO, spatial image cross-correlations (ICCS) were calculated and expressed as delta ICCS (TSPO/CD11b - TSPO/F4/80) for each imaging plane. Inserts show expression of markers in representative cross sections of $(\mathbf{E})$ mature plaque and $(\mathbf{F})$ fatty streak regions. 
Figure 4: Regions of Interest (ROIs) used in PET imaging of ${ }^{18}$ F-PBR111 aortic uptake. Axial (A, B), coronal (C, D) and sagittal (E, F) views of CT (A, C and E) and PET (B, D and F) images. The aortic ROI is shown in yellow and the lateral tail vein $\mathrm{ROI}$ is shown in red.

Figure 5: Quantification of ${ }^{18} \mathrm{~F}$-PBR111 uptake in thoracic aorta. (A) Time-activity curves calculated by subtracting the lateral tail vein $\mathrm{ROI}$ from the thoracic aorta $\mathrm{ROI}$ and (B) corresponding area under curve values. Values are mean \pm SD and expressed as percentage of injected dose per cubic $\mathrm{cm}\left(I D / \mathrm{cm}^{3}\right) \cdot \mathrm{n}=4-5 /$ group, ${ }^{*} \mathrm{p}<0.05$. 
A

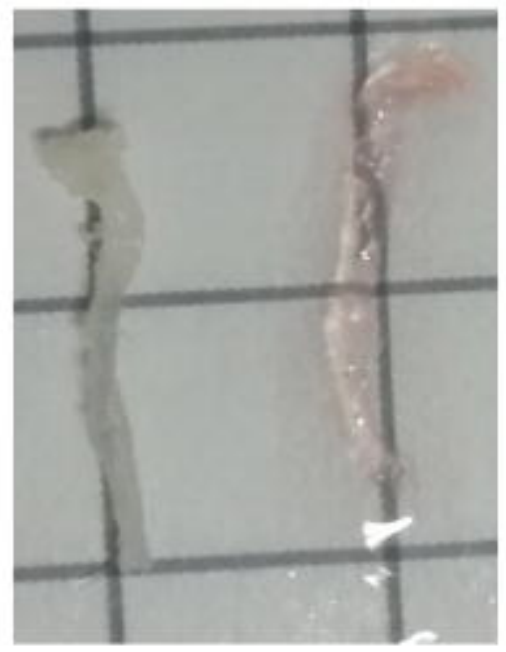

C

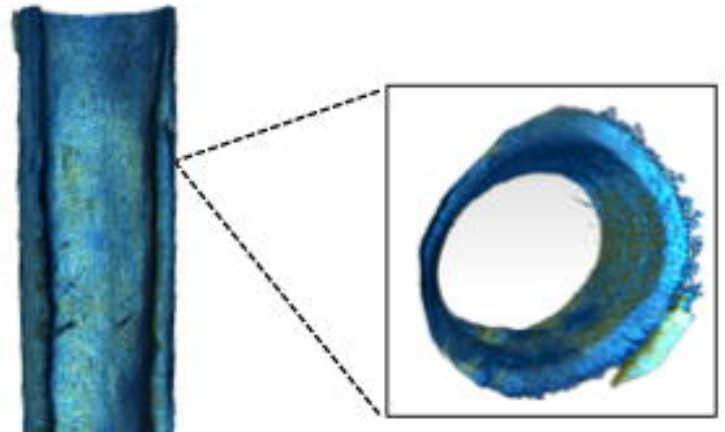

E

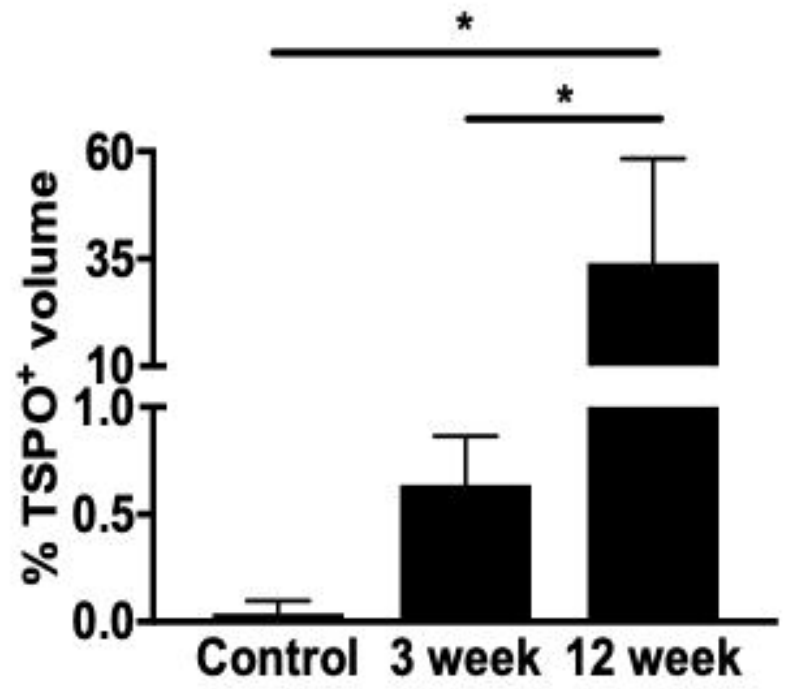

D
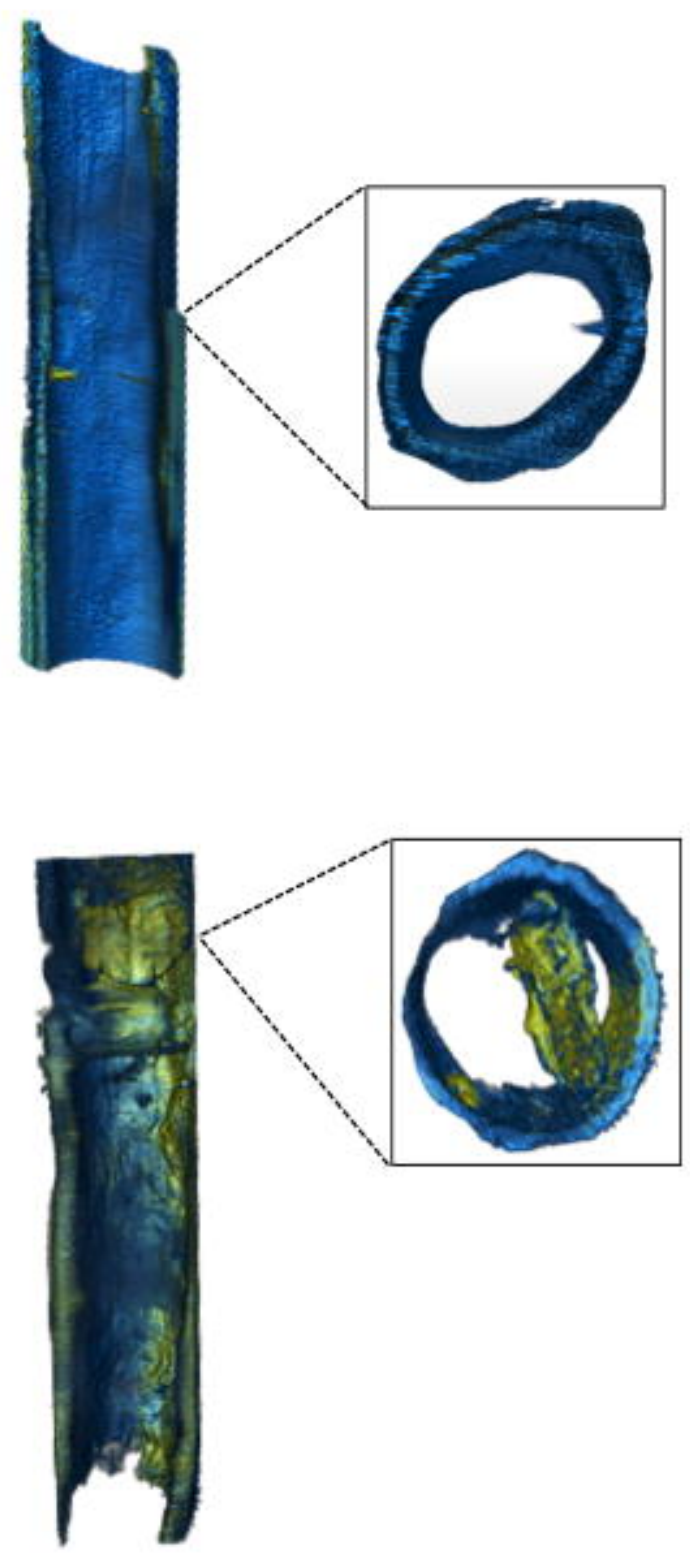

D 
A

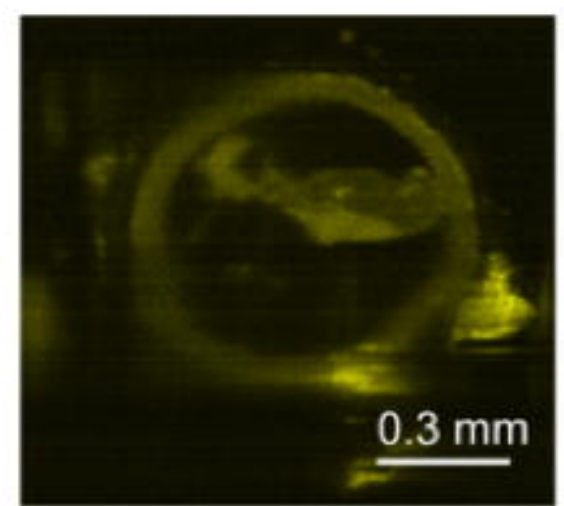

Before masking

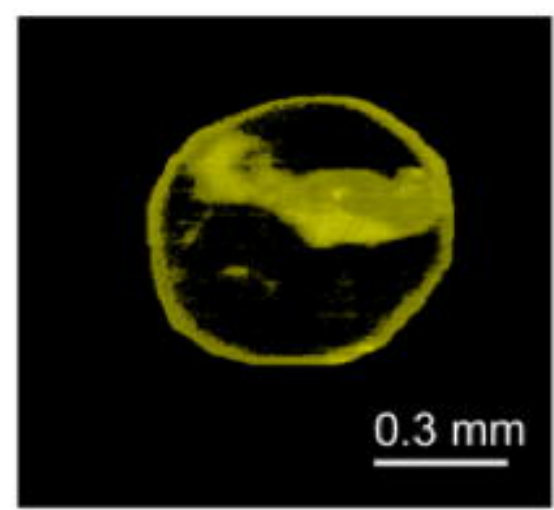

After masking

B

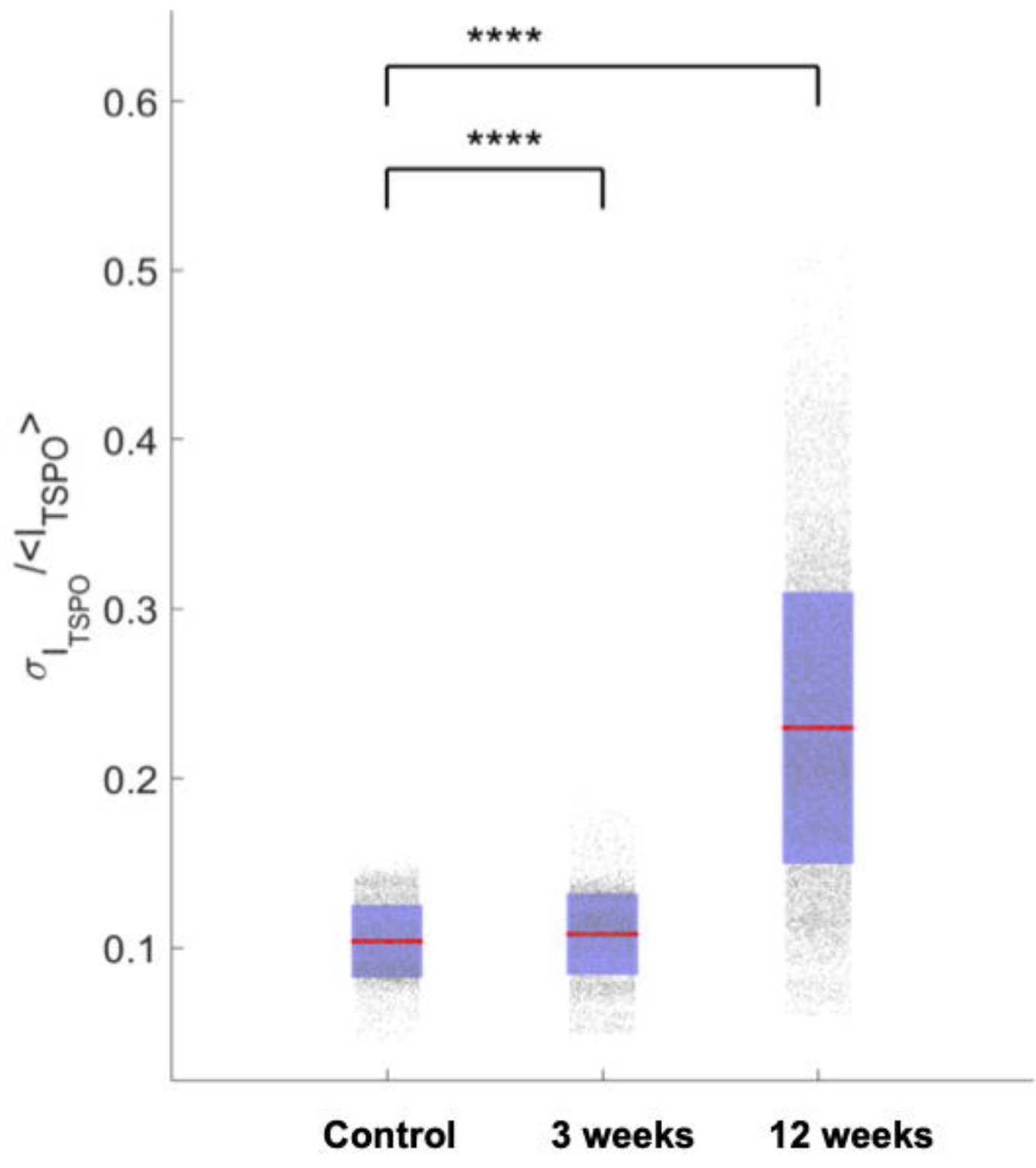


A
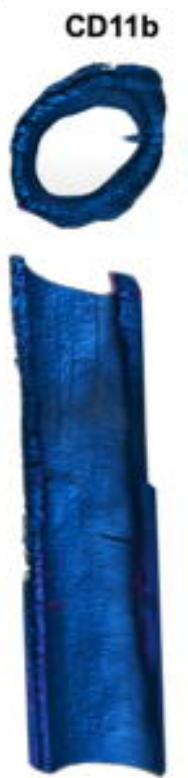

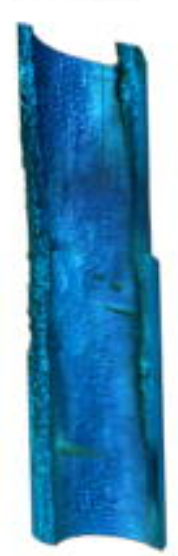

B

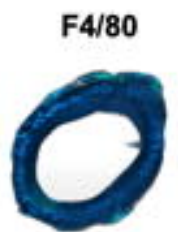

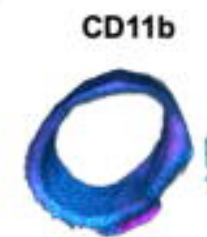

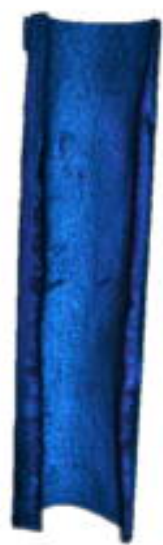

F $4 / 80$
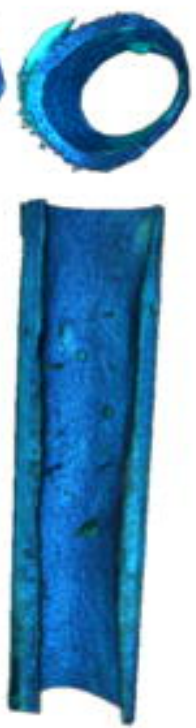

C
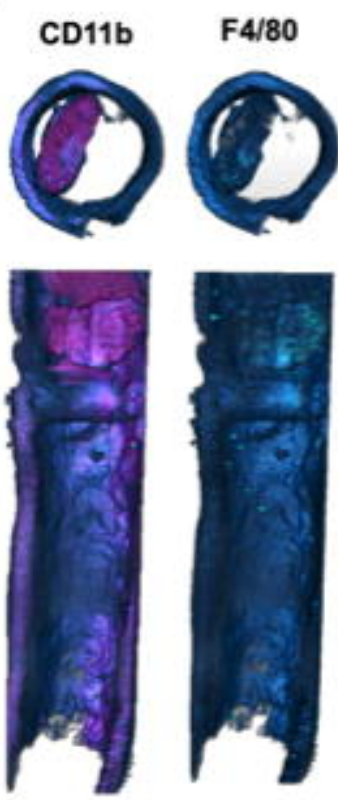

D

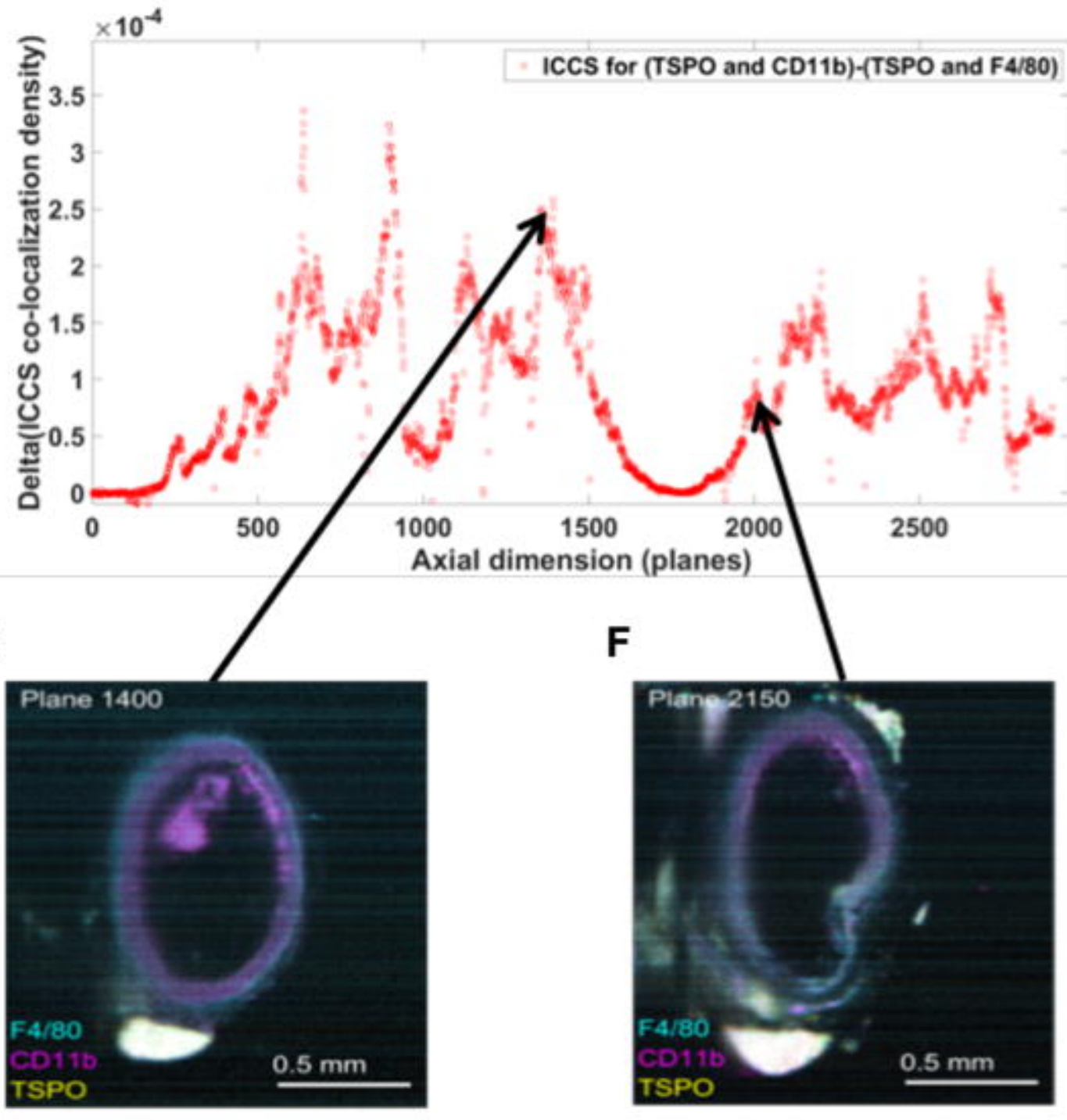


A

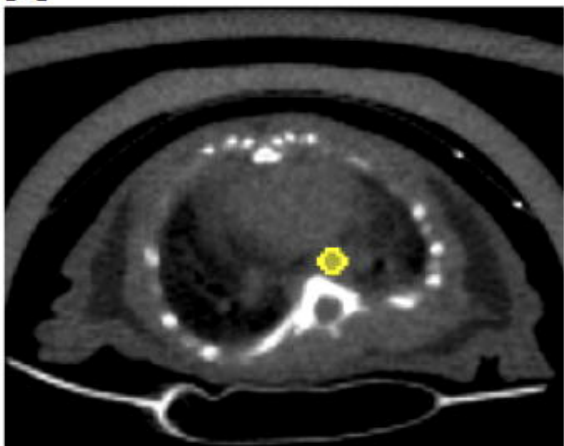

C

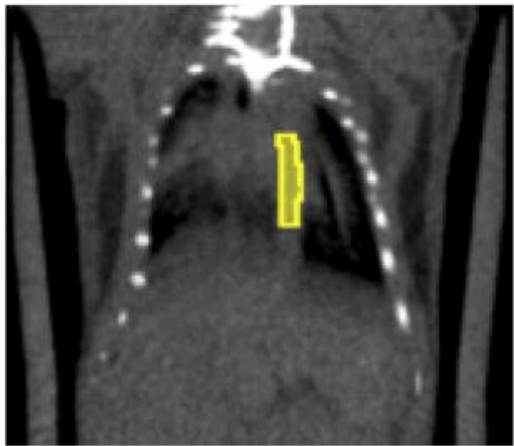

E

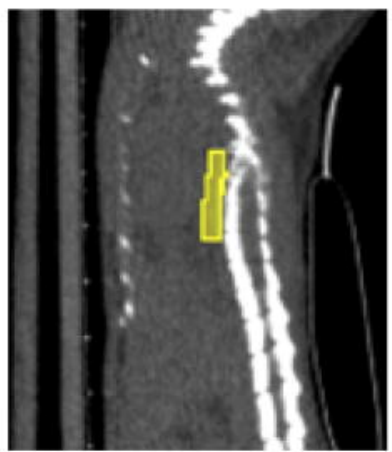

B

D

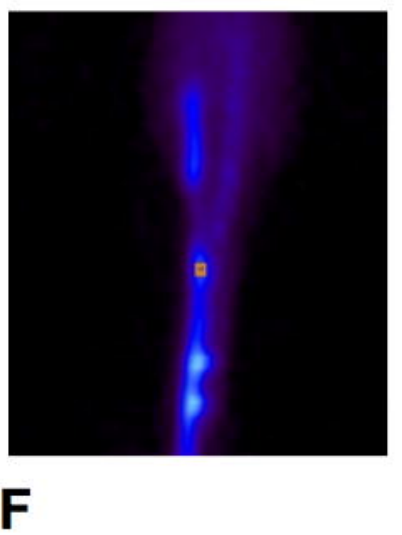



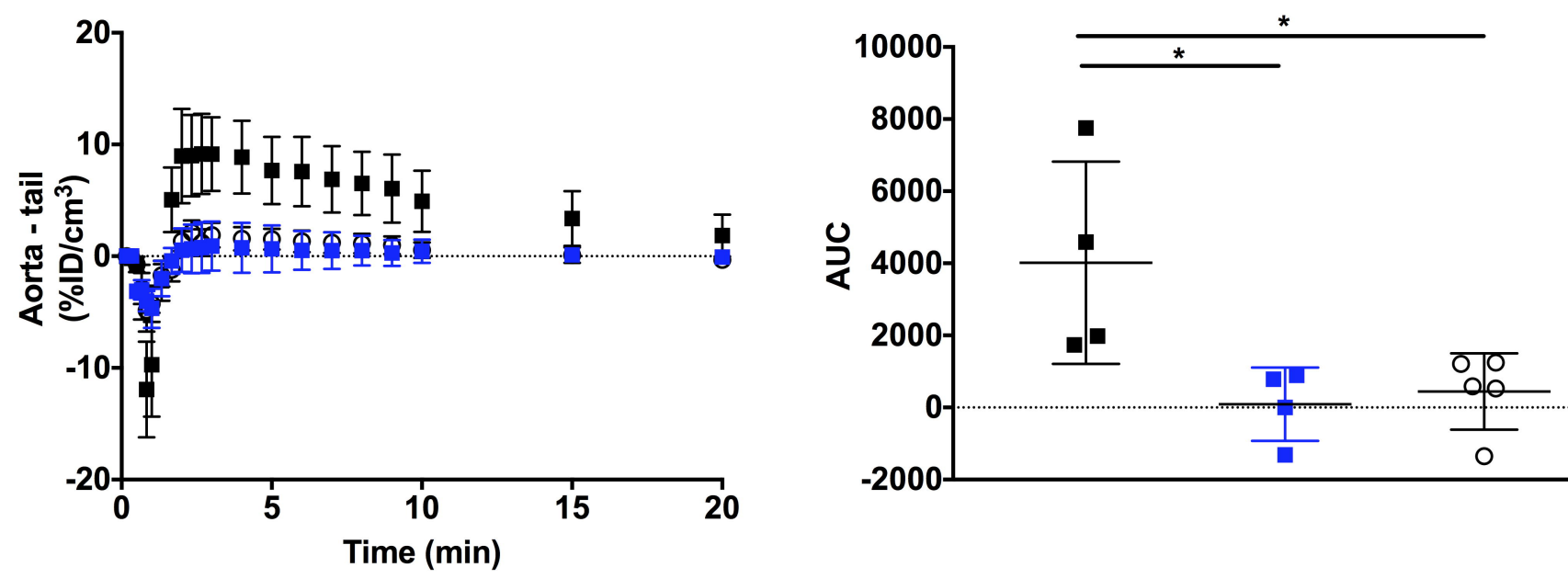

○ Control 3 week 12 week apoE-/- 